\title{
Comunicación
}

\section{FRECUENCIA DE PRESENTACIÓN DEL DESPLAZAMIENTO DE ABOMASO A LA IZQUIERDA EN VACAS LECHERAS DE LA CUENCA DE LIMA}

\author{
Frequency of Left Displacement of Abomasum in Dairy Cows in the Lima \\ REGION
}

David A. Silva G. ${ }^{1}$, Alfredo Delgado C. ${ }^{1,3}$, Roberto Evaristo R. ${ }^{1,4}$, Néstor Falcón P. ${ }^{2,4}$

\section{Resumen}

\begin{abstract}
El objetivo del presente trabajo fue determinar la frecuencia de casos de desplazamiento de abomaso a la izquierda (DAI) en 10 establos de la cuenca lechera de Lima en el periodo comprendido entre los años 2000 y 2004. Se encontró una frecuencia de ocurrencia de 6\%o (121 casos entre 20131 partos), donde el mayor número se presentó entre la $2^{\text {a }}$ y $4^{\mathrm{a}}$ semana postparto. El $74 \%$ de vacas afectadas fue enviado al camal.
\end{abstract}

Palabras clave: vacas, desplazamiento de abomaso a la izquierda, DAI, establo, lechería

\section{Abstract}

The objective of the present study was to determine the frequency of left displacement of the abomasum in dairy cows in 10 farms of the Lima region between years 2000 to 2004 . The frequency occurrence was 6\%o (121 cases within 20131 parturitions), where the largest number of cases occurring during the $2^{\text {nd }}$ and $4^{\text {th }}$ week after calving. Also, $74 \%$ of affected cows were sent to the slaughterhouse.

Key words: cow, left displaced abomasum, DA, barn, dairy

${ }^{1}$ Clínica de Animales Mayores, ${ }^{2}$ Laboratorio de Medicina Veterinaria Preventiva, Facultad de Medicina Veterinaria, Universidad Nacional Mayor de San Marcos, Lima

${ }^{2}$ E-mail: aldelgadoc@gmail.com

${ }^{4}$ Dirección actual: Facultad de Veterinaria y Zootecnia, Universidad Peruana Cayetano Heredia, Lima 
Cuadro 1. Frecuencia de casos de desplazamiento de abomaso a la izquierda (DAI) por cada mil vacas paridas por año en el periodo 2000-2004 en 10 establos de Lima

\begin{tabular}{cccc}
\hline Años & $\begin{array}{c}\text { Partos } \\
(\mathrm{n})\end{array}$ & $\begin{array}{c}\text { Casos de DAI } \\
(\mathrm{n})\end{array}$ & $\begin{array}{c}\text { Tasa por cada mil } \\
\text { vacas paridas }\end{array}$ \\
\hline 2000 & 4,017 & 8 & 2.0 \\
2001 & 3,969 & 41 & 10.3 \\
2002 & 3,992 & 29 & 7.3 \\
2003 & 4,018 & 16 & 4.0 \\
2004 & 4,135 & 27 & 6.5 \\
\hline Total & 20,131 & 121 & 6.0 \\
\hline
\end{tabular}

Cuadro 2. Frecuencia de ocurrencia de desplazamiento de abomaso a la izquierda (DAI) por cada mil vacas paridas por año en 10 establos lecheros de Lima (periodo 2000-2004)

\begin{tabular}{ccrrr}
\hline \multirow{2}{*}{ Establo } & $\begin{array}{c}\text { Prod. leche } \\
(\text { L/día })\end{array}$ & Partos & \multicolumn{2}{c}{ Casos DAI } \\
\cline { 4 - 5 } & 28.1 & 2,072 & $(\mathrm{n})$ & $(\% \circ)$ \\
\hline 1 & 20.0 & 308 & 30 & 14.5 \\
2 & 27.5 & 1,617 & 1 & 3.2 \\
3 & 19.0 & 3,433 & 18 & 11.1 \\
4 & 23.0 & 2,866 & 22 & 6.4 \\
5 & 23.1 & 4,126 & 2 & 0.7 \\
6 & 26.5 & 887 & 43 & 10.4 \\
7 & 23.0 & 2,072 & 5 & 5.6 \\
8 & 23.4 & 2,154 & 0 & 0 \\
9 & 26.0 & 596 & 0 & 0 \\
10 & & 20,131 & 0 & 0 \\
\hline Total & & & & 6.0 \\
\hline
\end{tabular}

La producción láctea bovina de la cuenca del valle Lima se incrementó de 82.2 a 179.7 mil TM entre 1993 y el 2001, sin que hubiese variaciones importantes en el número de animales (Webb y Fernández-Baca, 2002). Esta mejora productiva fue como respuesta de un mejor manejo de la nutrición y alimentación; sin embargo, había indicios que estos factores no estaban adecuadamente controlados debido a un incremento de enfermedades metabólicas, incluyendo un aumento inusual de la presentación del desplazamiento de abomaso a la izquierda (DAI) (Delgado, 2001).

El DAI es causado por factores nutricionales, tipo de alimentación y estado fisiológico del animal. Estos factores se con- 
jugan en la etapa de transición, donde la vaca pasa de una dieta rica en forraje (preparto) a una dieta rica en concentrado (postparto), permitiendo una mayor formación de ácidos grasos volátiles (AGV) y $\mathrm{NH}_{3}$ (Cuninghan, 1999).

El abomaso, durante la última fase de la gestación y el proceso del parto, es desplazado en dirección craneoventral por el útero grávido; además, en el postparto, muchas vacas pasan por un proceso de hipocalcemia subclínica (Cuninghan, 1999) y disminución de la contractibilidad del abomaso por efecto de los AGV. Estos factores pueden impedir que el abomaso regrese a su posición normal, quedando atrapado debajo del rumen y apareciendo entre la luz y la pared abdominal (Merck, 2000). Generalmente, el DAI se presenta en vacas lecheras adultas de gran tamaño y alta producción, principalmente entre el parto y los dos meses postparto; sin embargo, se describe que el $64 \%$ de los casos se presentan durante la primera semana postparto (Rebhum, 1999). Por otro lado, hay reportes que lo relacionan con los meses de mayor ocurrencia de partos (Jiménez et al., 1993).

El DAI tiene una repercusión económica por la disminución de la producción de leche, pago por servicios veterinarios, cuidados post-operatorios, medicamentos, e incluso por pérdidas de animales que deben ser descartadas del rebaño (Van Suan, 2000). El presente estudio retrospectivo se hizo para determinar la frecuencia de presentación del DAI en la cuenca lechera de Lima entre el 2000 y el 2004. Para este efecto, se recolectó información en 10 establos ubicados en Lurín, Huacho, Chancay, Puente Piedra, Carabayllo y Callao. Se recabó datos sobre los animales, producción de leche, aspectos reproductivos, alimentación y ocurrencia de enfermedades metabólicas, especialmente durante el periodo de transición.

Se encontró 121 casos de DAI en los 5 años en estudio, pero con un comportamiento inusual, toda vez que hubo pocos casos en el 2000, y un marcado incremento en el 2001, para luego mantenerse en un nivel intermedio en los años siguientes (Cuadro 1). Es posible que esta enfermedad, de relativa poca importancia hasta el año 2000, haya pasado desapercibida para los clínicos, pero a partir del reporte de Delgado (2001) en el año siguiente, se pudo haber prestado mayor atención a la presentación de esta patología.

Tres establos no tuvieron ocurrencias de DAI (establos 8, 9 y 10), en tanto que los establos 1,3 y 6 presentaron frecuencias de 10.4, 11.1 y $14.5 \%$, respectivamente (Cuadro 2), sin que se observase una relación de importancia con la producción de leche, número de parto o dieta de transición. La única vaca que llegó a presentar DAI en el establo 2 parió mellizos, lo cual la hace más propensa a presentar esta enfermedad (Breukink, 1991).

La mayor presentación de casos de DAI se presentó entre la segunda y cuarta semana del parto (65.3\% de los casos) y muy pocos ocurrencias después de la octava semana, como lo señala Rebhum (1999). Es posible que muchas de las vacas con DAI hayan estado con hipocalcemia subclínica (Meléndez et al., 2003), sin que se las haya detectado, haciéndose más propensas a sufrir DAI que vacas con hipocalcemia clínica (Goff y Horst, 1993; Masey et al., 1993).

Un elevado número de vacas con DAI fueron destinadas al camal (74\%), en tanto que el resto se recuperaron, sea con tratamiento conservativo o quirúrgico. Es posible evitar esta alta tasa de descarte de animales afectados por DAI con una adecuada prevención y tratamiento quirúrgico oportuno.

\section{Literatura Citada}

1. Andresen H. 2001. Vacas secas y en transición. Rev Inv Vet, Peru 12(2): 36- 49.

2. Breukink HJ. 1991. Abomasal displacement, etiology, pathogenesis treatment and prevention. Bovine Practitioner 26: 148-153. 
3. Cunninghan J. 1999. Fisiología veterinaria. $2^{\mathrm{a}}$ ed. México DF: McGraw-Hill Interamericana. $763 \mathrm{p}$.

4. Delgado A. 2001. Presentación inusual de desplazamiento de abomaso a la izquierda. Rev Inv Vet, Perú 12(2): 138-141.

5. Goff JP, Horst RL. 1993. Oral administration of calcium salts for treatment of hypocalcemia in cattle. $\mathrm{J}$ Dairy Sci 76: 101-108.

6. Jiménez F, Brandau G, Tesouro M, Josa J, De Gaspar I, Brandau D. 1993. Síndrome de dilatación y torsión del abomaso. Aula Vet Bovis 52: 11-30.

7. Massey CD, Wang C, Donovan GA.1993. Hypocalcemia at parturition as a risk factor for left displacement of the abomasum in dairy cows. J Am Vet Med Assoc 203: 852-853.
8. Melendez P, Donovan GA, Risco CA, Littell R, Goff JP. 2003. Effect of calcium-energy supplements on calvingrelated disorders, fertility and milk yield during the transition period in cows fed anionic diets. Theriogenology 60: 843854.

9. Merck. 2000. El manual Merck veterinaria. $5^{\text {ta }}$ ed. Barcelona: Océano. 2558 p.

10. Rebhum W. 1999. Enfermedades del ganado vacuno lechero. Zaragoza: Acribia. 678 p.

11. Van Saun RJ. 2000. Periodo de transición: Consecuencias sanitarias y reproductivas. [Internet]. Disponible en: http://www.providesa.com/seprjul1 htm

12. Webb R, Fernández-Baca G. 2002. Anuario Perú en números. Lima: Ed. Cuanto. $1320 \mathrm{p}$. 